\title{
Inferior vena cava injury caused by an anteriorly migrated cage resulting in ligation: case report
}

\author{
Dai Ariyoshi, MD, Shigeo Sano, MD, PhD, and Naohiro Kawamura, MD, PhD \\ Department of Orthopaedic Surgery, Sanraku Hospital, Tokyo, Japan
}

Anterior dislodgement of the transforaminal lumbar interbody fusion (TLIF) cage is one of the severe complications seen in this procedure, which may cause an intraoperative major vessel injury. The objective of this report is to present a rare case of inferior vena cava (IVC) injury during revision surgery for removal of the anteriorly migrated cage.

The authors describe a case of 74-year-old woman with lumbar spinal canal stenosis and degenerative scoliosis. During the TLIF surgery, an inserted titanium cage at the L4-5 level dislodged anteriorly to the retroperitoneal space without massive bleeding from the disc space. In the second surgery, which was performed via an anterior retroperitoneal approach to remove the migrated cage, massive torrential bleeding occurred because of IVC injury. The laceration in the posterior wall of the IVC necessitated ligation of this vessel and both common iliac veins by a vascular surgeon. Postoperative edema of the lower extremities after ligation of the vessels was well tolerated, and the patient showed almost full recovery.

For removal surgery of an anteriorly migrated cage, the surgeon should be well prepared for the risk of IVC injury, including requesting the attendance of a vascular surgeon. Ligation of the infrarenal IVC is an acceptable solution in irreparable IVC injury.

http://thejns.org/doi/abs/10.3171/2015.6.SPINE1544

KEY WORDS cage migration; ligation; inferior vena cava; common iliac veins; vascular injury; posterior lumbar spinal surgery; lumbar

A LTHOUGH major vessel injury after spine surgery is an infrequent complication, it is associated with a high mortality rate. We report a case with laceration of the inferior vena cava (IVC), which caused massive torrential bleeding during removal of an anteriorly migrated cage. Ligation of the IVC was necessary for rescue.

\section{Case Report}

History and Examination

A 74-year-old woman presented at our hospital with severe low-back pain and intermittent claudication. Conservative therapies of several kinds were done at another hospital, but they were ineffective. Radiographs and MRI studies showed multiple stenoses and degenerative scoliosis (Figs. 1 and 2).

\section{First Operation}

In June 2010, a posterior decompression, correction, and fusion surgery was performed using the corrective transfo- raminal lumbar interbody fusion (TLIF) technique. After pedicle screw insertion and decompression, the right L4-5 disc space was shaved via a transforaminal approach. Maximal opening of the right L4-5 disc space was obtained using our special spreader. A 12-mm titanium cage was filled with autologous iliac bone graft and was tapped into the disc space. Because of obscured vision with blood in the field, monotonous shape, and multiple similar scale lines of the introducer, the operator could not keep the depth under control and tapped the cage to an excessive depth. After taking off the introducer, a radiograph was taken. It showed the cage passing anteriorly through the anulus to the retroperitoneal space (Fig. 3). After several vain attempts to capture the cage with its introducer, the surgeon decided to remove it via an anterior approach on another day because no massive bleeding or change of vital signs occurred. Another cage was inserted meticulously into the same L4-5 disc space, and the corrective TLIF was done at left L2-3 and left L3-4 with two additional cages, followed by posterolateral fusion and rod fixation (Fig. 4).

ABBREVIATIONS IVC = inferior vena cava; TLIF = transforaminal lumbar interbody fusion. ACCOMPANYING EDITORIAL See pp 407-408. DOI: 10.3171/2015.7.SPINE15688.

SUBMITTED January 11, 2015. ACCEPTED June 11, 2015.

INCLUDE WHEN CITING Published online December 4, 2015; DOI: 10.3171/2015.6.SPINE1544. 


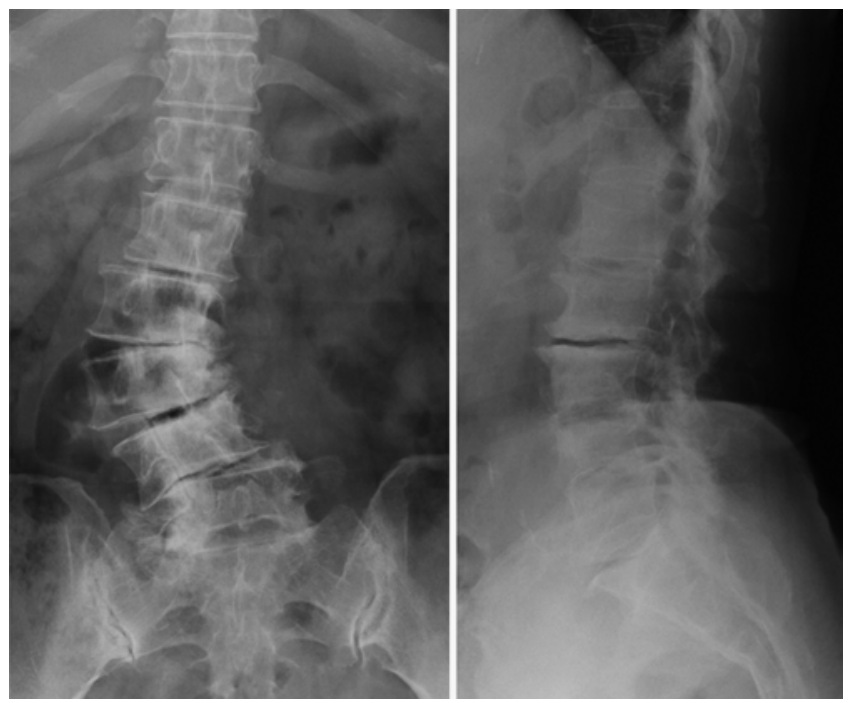

FIG. 1. Plain radiographs showing degenerative scoliosis in anteroposterior (left) and lateral (right) views.

\section{Initial Postoperative Course}

After the operation the patient rested in bed, in good general condition. Enhanced CT scans showed that the anteriorly migrated cage placed in front of the L4-5 disc verged on the IVC and abdominal aorta. No hematoma was observed around the cage (Fig. 5).

\section{Second Operation}

On postoperative Day 7, surgery to remove the migrated cage was performed. The L4-5 region was accessed through a right retroperitoneal approach. The cage was identified easily; it was pulled carefully and gently. When the cage was lifted slightly, bleeding started gradually but

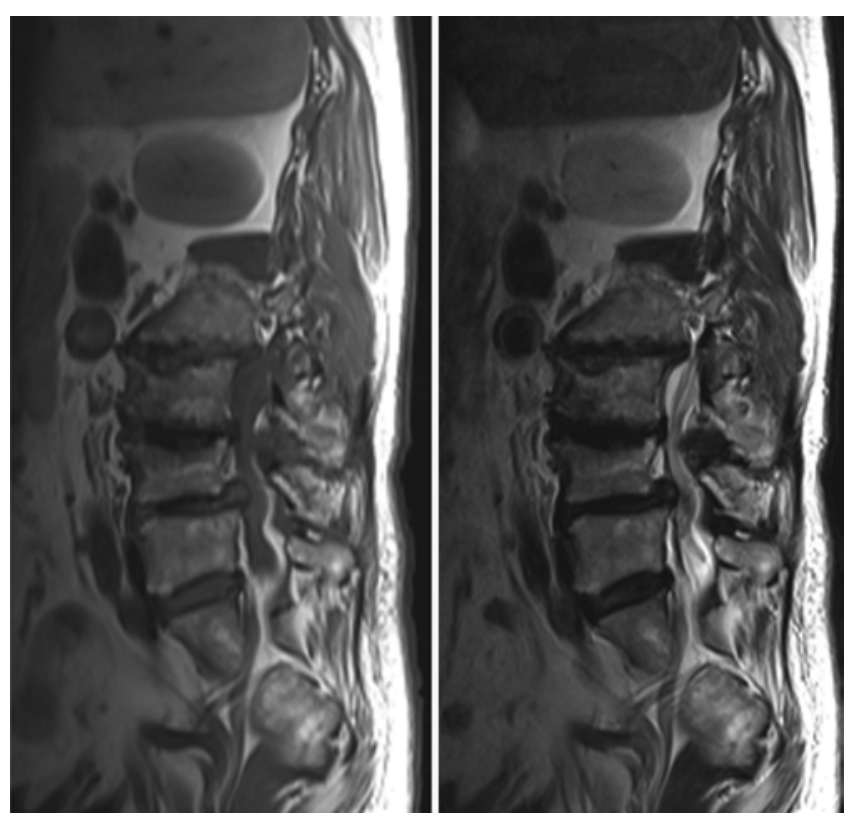

FIG. 2. T1-weighted (left) and T2-weighted (right) sagittal images showing the multiple stenoses at L3-5.

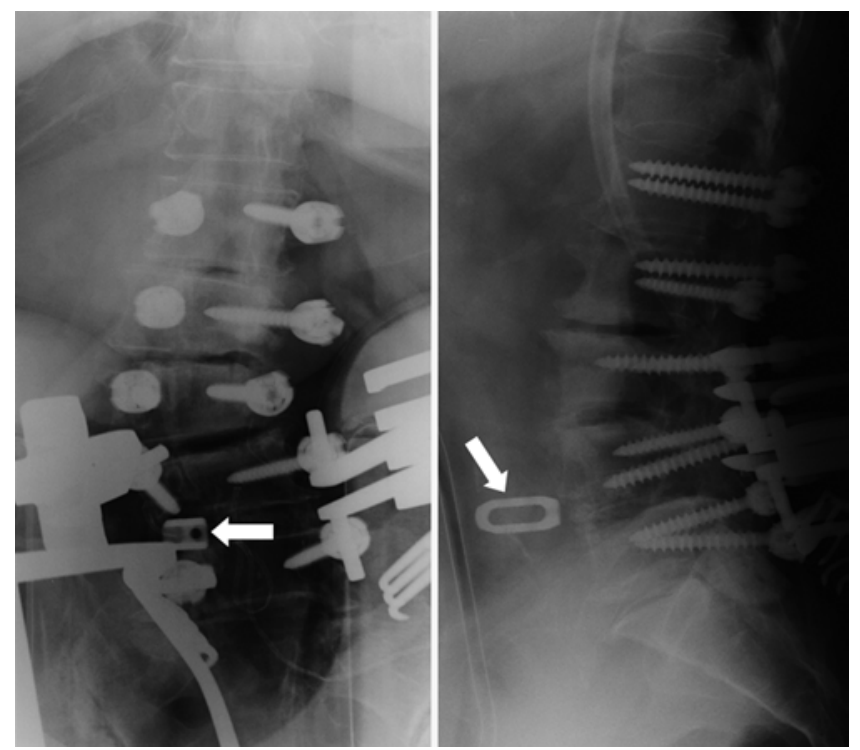

FIG. 3. Intraoperative plain radiographs showing the anteriorly migrated cage (arrows) in anteroposterior (left) and lateral (right) views.

strongly. We suspected IVC injury and decided to remove the cage and repair the vein. Because the tear was located on the back side of the IVC, it seemed difficult to repair. We called a vascular surgeon for assistance.

Emergency laparotomy was performed through another median incision, which revealed that the perforation of the IVC was immediately adjacent to both common iliac veins. The aorta and both iliac arteries were taped for temporary tightening to reduce bleeding from the injured vein. The resultant defect over the posterior aspect of IVC could not be repaired, primarily because of its size and location. Blood pressure dropped to a degree that was

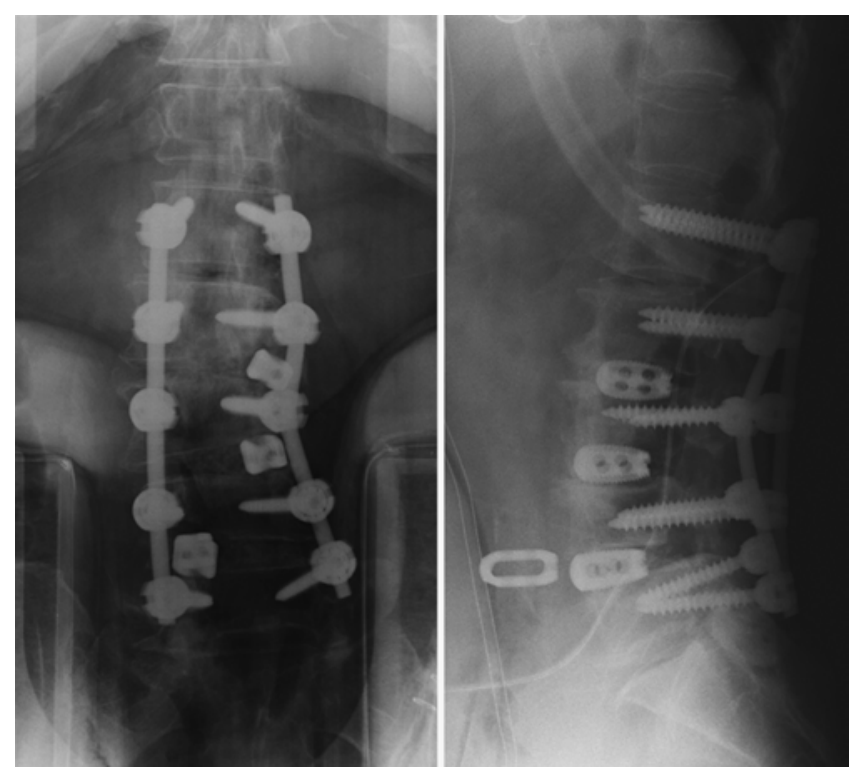

FIG. 4. Plain radiographs after the first operation showing the anteroposterior (left) and lateral (right) views. The anteriorly migrated cage is located in front of the properly placed L4-5 cage. 

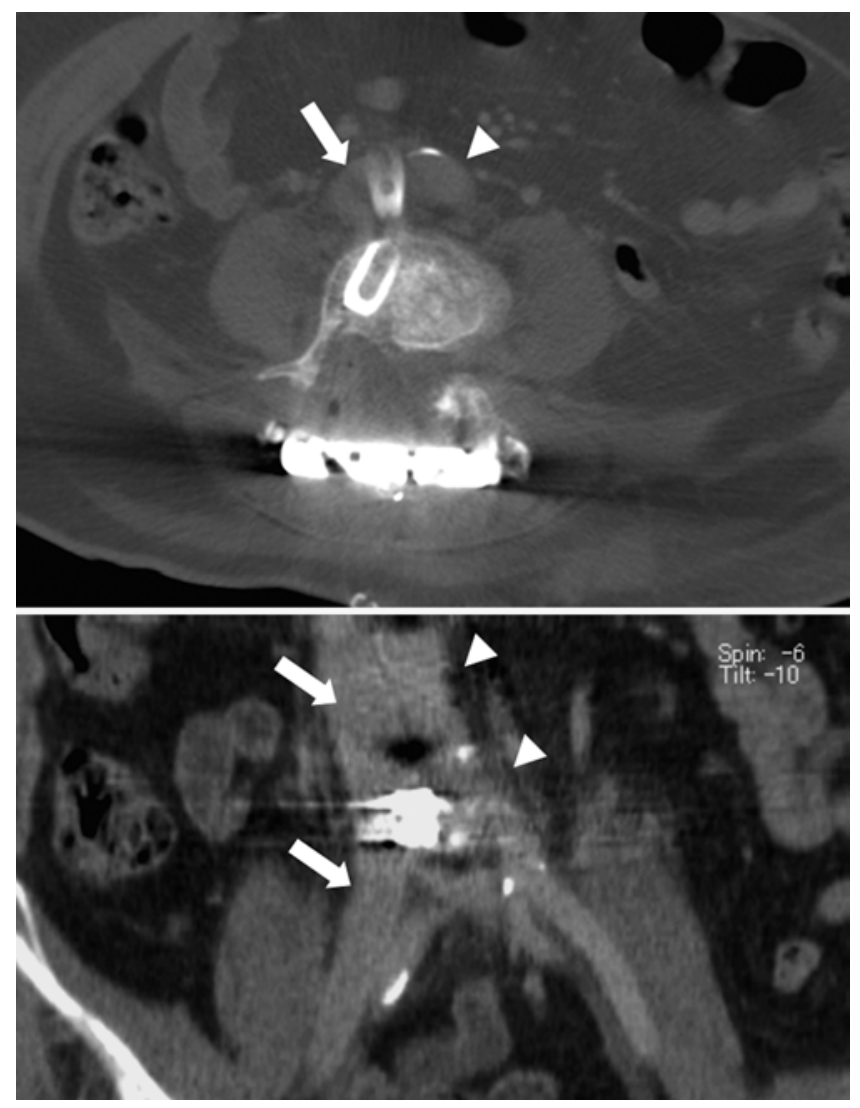

FIG. 5. Enhanced CT image in axial (upper) and coronal (lower) views showing the anteriorly migrated cage placed at the L4-5 level, which had penetrated the anterior longitudinal ligament and had become adherent to the IVC (arrows) and abdominal aorta (arrowheads). Bleeding was not observed around the cage.

not measurable using a noninvasive device. Aggressive resuscitation with blood transfusion and constant infusion of vasopressor agents such as epinephrine and dopamine were continued. Finally, the vascular surgeon decided to ligate the IVC and both common iliac veins for rescue. After ligation, the vital parameters became stable. Meticulous hemostasis was performed for another hour to stop the oozing blood. She received a total of $90 \mathrm{U}$ of packed red blood cells, $70 \mathrm{U}$ of fresh frozen plasma, and $60 \mathrm{U}$ of fresh platelets. The estimated total blood loss was approximately $20,000 \mathrm{ml}$.

\section{Second Postoperative Course}

After the operation, the patient was intensively treated with a respirator and massive postoperative blood transfusion. Her general condition improved gradually. Although temporary pulmonary edema and anasarca occurred, the endotracheal tube was extubated smoothly on the 5th day after the second operation. Postoperative edema of the lower extremities was well tolerated (Fig. 6), and preoperative deformity was well corrected (Fig. 7). When she was discharged 3 months after the second operation she was walking without any assistance, and her ambulation ability showed significant improvement in 1 year compared with her preoperative status. Although no further analysis

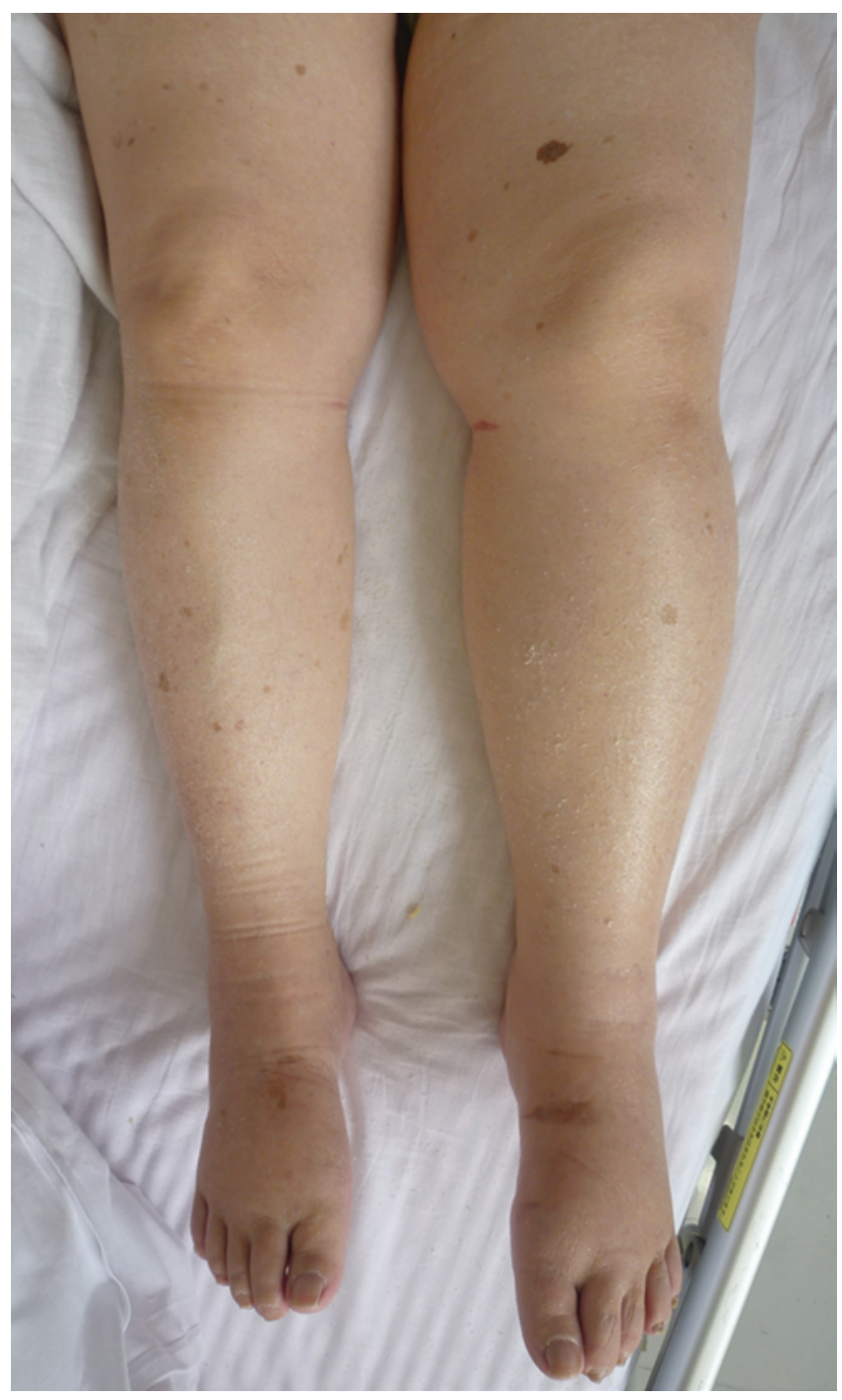

FIG. 6. Photograph showing the edema of lower extremities after ligation of the IVC. Figure is available in color online only.

of deep venous thrombosis was done, we found no circulation problems with continuous warfarin administration. Solid bony fusion of TLIF segments was confirmed by radiograph at 1.5 years postoperatively.

\section{Discussion}

With the advent of spinal instrumentation of various kinds, the number of anterior lumbar interbody fusion (ALIF), posterior lumbar interbody fusion (PLIF), and TLIF procedures, as effective and safe treatment for various lumbar spinal diseases, has been increasing. Some reports ${ }^{1,2,4-6,9}$ describe the existence of severe vascular complications resulting from these procedures.

As described in this paper, we reported a rare case of IVC ligation during surgery for removal of an anteriorly migrated TLIF cage. This experience provided an important lesson: we should consider the risk of major vessel injury by a migrated cage, even without obvious massive bleeding. In addition, we recognized the importance 


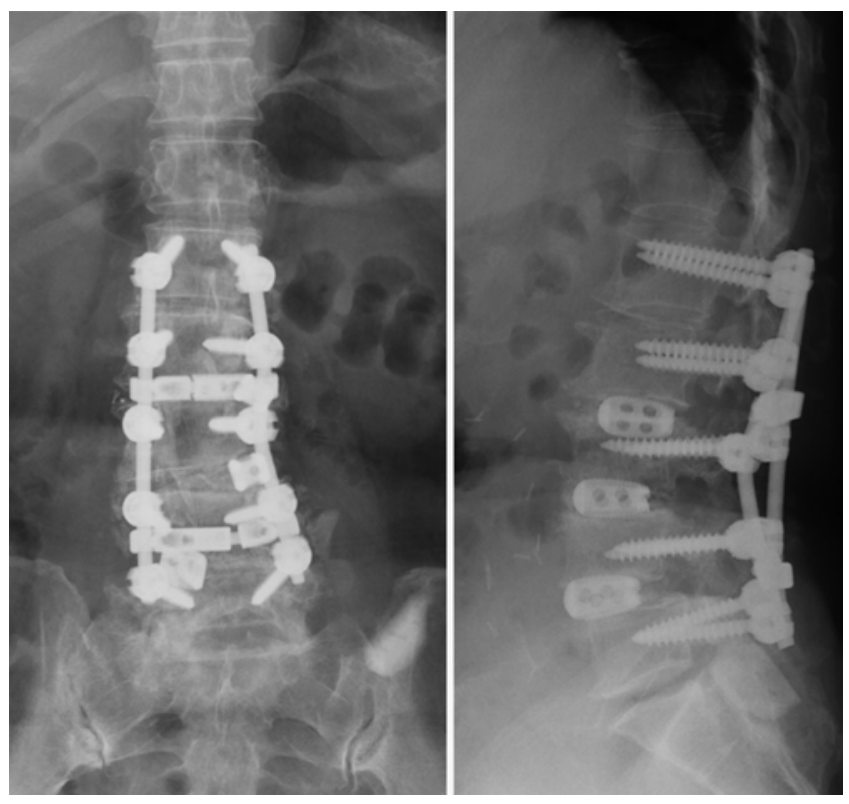

FIG. 7. Plain radiographs after the second operation showing the wellaligned spine in anteroposterior (left) and lateral (right) views.

of having a vascular surgeon not only available, but also helping with the anterior portion in this kind of procedure from the very beginning.

In our case, ligations of the IVC and the common iliac veins were unavoidable. They presented an acceptable solution to a major disruption of these veins that might otherwise have required several hours to repair, in addition to the necessity of multiple blood transfusions. Although we were not aware of it, these ligations have been performed before the introduction of an IVC filter for the control of recurrent pulmonary emboli in instances in which anticoagulant therapy had failed. The procedure was not associated with surgery-related death or further embolism. Although early postoperative leg edema was common, later leg complications proved to be minor if the legs were carefully managed. . $^{3,10}$

For ligations of the IVC, whether done proximal to the renal vein or distal to it, the level of ligation is an important determinant of resultant death. Injury to the IVC as a result of trauma has a high mortality rate. Nevertheless, some survivors of ligation of infrarenal IVC performed as a rescue operation do exist. Moreover, none of the survivors had significant long-term edema or extremity dysfunction. In contrast, ligation of the suprarenal IVC has few survivors. ${ }^{7,8}$ Consequently, ligation of the infrarenal IVC might be an acceptable damage control procedure, in cases presenting no realistic alternative for preservation of the IVC. Ligation of the suprarenal IVC should be avoided to the greatest degree possible.

\section{Conclusions}

For removal of an anteriorly migrated cage, the surgeon should be well prepared for the risk of IVC injury, including requesting the attendance of a vascular surgeon. Ligation of the infrarenal IVC is an acceptable solution in irreparable IVC injury.

\section{Acknowledgments}

We thank Dr. H. Tsubata for his great support in perioperative intensive care of this patient.

\section{References}

1. Baker JK, Reardon PR, Reardon MJ, Heggeness MH: Vascular injury in anterior lumbar surgery. Spine (Phila Pa 1976) 18:2227-2230, 1993

2. Brau SA, Delamarter RB, Schiffman ML, Williams LA, Watkins RG: Vascular injury during anterior lumbar surgery. Spine J 4:409-412, 2004

3. Dale WA: Ligation of the inferior vena cava for thromboembolism. Surgery 43:24-44, 1958

4. Fantini GA, Pappou IP, Girardi FP, Sandhu HS, Cammisa FP Jr: Major vascular injury during anterior lumbar spinal surgery: incidence, risk factors, and management. Spine (Phila Pa 1976) 32:2751-2758, 2007

5. Pawar UM, Kundnani V, Nene A: Major vessel injury with cage migration: surgical complication in a case of spondylodiscitis. Spine (Phila Pa 1976) 35:E663-E666, 2010

6. Shih PY, Lau HP, Jeng CS, Hung MH, Chan KC, Cheng YJ: Iatrogenic left internal iliac artery perforation during lumbar discectomy. Acta Anaesthesiol Taiwan 47:196-199, 2009

7. Sullivan PS, Dente CJ, Patel S, Carmichael M, Srinivasan JK, Wyrzykowski AD, et al: Outcome of ligation of the inferior vena cava in the modern era. Am J Surg 199:500-506, 2010

8. Votanopoulos KI, Welsh FJ, Mattox KL: Suprarenal inferior vena cava ligation: a rare survivor. J Trauma 67:E179-E180, 2009

9. Wang YR, Liu YC, Chung NC, Wu SZ, Chen MS, Ye XD, et al: Inferior vena cava tear during posterior spinal fusion surgery. Acta Anaesthesiol Sin 41:89-92, 2003

10. Zoeckler SJ: Inferior vena cava ligation. J Iowa State Med Soc 50:552-556, 1960

\section{Disclosures}

The authors report no conflict of interest concerning the materials or methods used in this study or the findings specified in this paper.

\section{Author Contributions}

Conception and design: Sano. Acquisition of data: Ariyoshi. Drafting the article: Ariyoshi, Kawamura. Critically revising the article: Sano, Kawamura. Reviewed submitted version of manuscript: all authors. Study supervision: Sano, Kawamura.

\section{Correspondence}

Dai Ariyoshi, Department of Orthopaedic Surgery, Sanraku Hospital, 2-5 Kanda Surugadai, Chiyoda-ku, Tokyo 101-8326, Japan. email: jrprq017@yahoo.co.jp. 